Eur. J. Clin. Chem. Clin. Biochem.

Vol. 32, 1994, pp. 177-178

(c) 1994 Walter de Gruyter \& Co.

Berlin $\cdot$ New York

\title{
Combining Laboratory Tests for Diagnostic Decisions
}

\author{
By B. Schneider \\ Institut für Biometrie, Medizinische Hochschule Hannover, Hannover, Germany
}

(Received October 4, 1993)

Comment to the Paper

\section{Performance Assessment of Coupled Tests: The Effects of Statistical Non-Independence}

by A. Chiecchio, R. Malvano, F. Giglioli and A. Bo, this J. 32 (1994) 169-175

Summary: For the combination of two diagnostic tests often the $\mathrm{AND}$ and OR procedure is used. The AND procedure classifies a case as pathologic, if both tests classify it as pathologic; the OR procedure, if at least one test classifies it as pathologic. It is shown that these are no good combination procedures resulting in diagnostic qualities which can be even worse than the worst single test. Instead optimal multivariate statistical classification procedures should be used which give the best possible improvement of diagnostic quality.

\section{Introduction}

In their paper Chiecchio et al. (1) consider combinations of the results of two laboratory tests for diagnostic decisions according to the AND and OR rule. With the AND rule a case is classified as pathologic if both tests classify this case as pathologic. With the $O R$ rule a case is classified as pathologic if at least one of the two tests classifies the case as pathologic. In the paper the influence on sensitivity and specificity of the correlation between both test results is analysed by mathematical models and empirical data. This question is treated well and it is shown that the correlation may seriously influence the predictive values. But the fundamental question is whether the AND and OR combinations are good procedures and can be recommended.

\section{Discussion}

The advantage of both these combinations is their simplicity. One needs only know the classifications of the single tests to be able to classify immediately a case according to the combined rule. But both rules cannot be assumed to be "good" rules and they are often far from optimal. This is illustrated by a hypothetical example.

In figure 1 the test results of 10 non-pathologic and 10 pathologic cases for two hypothetical tests (x-test and y-test) are plotted in a $x-y$ coordinate system. It is assumed that for both tests the cutpoint is 5 . The empirical sensitivity for both tests is $70 \%$ and the speci-

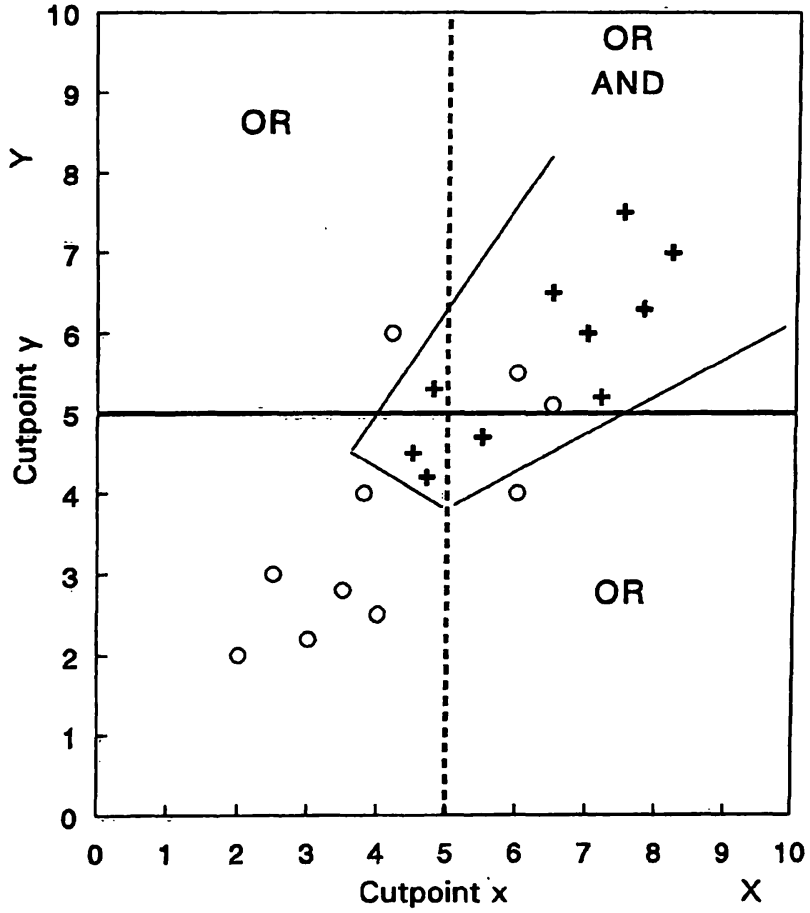

Fig. 1 Combination of diagnostic tests (hypothetical example) $\begin{array}{ll}\text { cutpoint } x & --- \text { cutpoint } y \\ O=\text { non-pathologic } & +=\text { pathologic }\end{array}$

ficity is also $70 \%$. According to Youden (2) the quality of a diagnostic procedure (for classification in two groups; e. g. pathologic and non-pathologic) can be expressed by the index $\mathrm{J}$ which is the sum of the sensitivity and the specificity minus $100 \%$. For both tests index $\mathrm{J}$ is $40 \%$. 
With the AND combination sensitivity decreases to $60 \%$ and specificity increases to $80 \%$. The quality index $\mathrm{J}$ is unchanged (40\%). With the OR combination sensitivity increases to $80 \%$ and specificity decreases to $60 \%$. The quality index $J$ remains at $40 \%$. In this hypothetical example neither the AND nor the OR combination improve the test quality procedures as compared with a single. This means that the combinations have no advantage over a single test. That similar situations arise empirically is demonstrated by the example given by Chiecchio et al (1). Here euthyroids and hypothyroids are diagnosed with T3 (triiodothyronine) as one test and fT3 (free triiodothyronine) as a second test. The values for sensitivity and specificity reported in the paper are: for sensitivity $67 \%$ (T3) and $78 \%$ (fT3), for specificity $95 \%$ (both tests). This results in a quality index $\mathrm{J}$ of $67 \%$ for T3 and $73 \%$ for $\mathrm{fT} 3$. The AND combination has (according to table 1 , case $C$ in l. c. (1)) an experimental sensitivity of $62 \%$ and a specificity of $99 \%$ resulting in $\mathrm{J}=61 \%$. This combination has a worse quality than both single tests. For the OR combination the experimental sensitivity is $83 \%$ and the specificity $91 \%$ resulting in $\mathrm{J}=74 \%$. The improvement of $\mathbf{J}$ (compared with fT3) is only marginal.

These examples show that AND and OR combinations cannot be recommended. There may be even a loss in diagnostic quality compared with the single tests.

Better and even optimal combinations of diagnostic tests can be obtained by multivariate statistical classification methods (see e. g. Schneider (3)). Instead of single cutpoints multivariate classification contours in the space of the combined test results are used in these methods. By these contours the space of the combined test results is divided into as many separate regions as there are diagnostic classes under constellation (in the case of two classes (pathologic and non-pathologic) in two regions). There are various statistical methods for constructing classification contours for optimal classification. The commonly used procedure is linear discriminant analysis which is adequate for combined test results with multivariate normal distribution and a common covariance matrix. For different covariance matrices quadratic discriminant analysis can be used. If there are doubts as to the normality assumption, non-parametric procedures such as the nearest neighbour procedure is recommended (see l.c. (3)). For the data of figure 1 such a nearest neighbour procedure was applied to get optimal classification for the combination of $x$-test and $y$-test. The classification contour is indicated by the polygonal curve in the $x-y$ plane. As can be seen from figure 1 the sensitivity of this optimal procedure is $100 \%$ and specificity $80 \%$, resulting in a quality index $\mathrm{J}=80 \%$. By this optimal combination the diagnostic quality of the single tests (and of the AND and OR combinations) is doubled. Table 1 gives the positive and negative predictive values of the single tests and the combinations for different prevalence values (instead of the prevalence the reciprocal value (i. e. the ratio non-pathologic cases/ pathologic cases) is tabulated). It can be seen that these predictive values as well are noticably improved by the optimal combination.
Tab. 1 Predictive valucs for single tests and combinations.

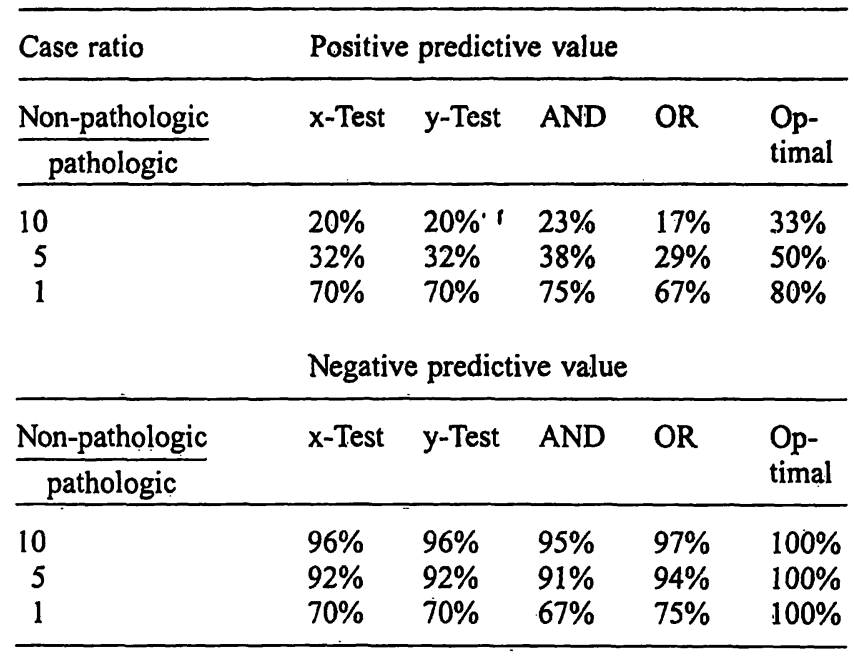

\section{Conclusion}

For the combination of diagnostic tests optimal statistical classification procedures should be used instead of the simple AND and OR combination. By these methods the diagnostic quality can be improved considerably, whereas by AND and OR combinations it can happen that no or a most marginal improvement is achieved.

\section{References}

1. Chiecchio A., Malvano, R., Giglioli, F. \& Bo, A. (1994) Performance assessment of coupled tests: The effects of statistical non-independence. Eur. J. Clin. Chem. Clin. Biochem. 32, 169-175.

2. Youden, W. J. (1950) Index for rating diagnostic tests. Cancer $3,32-35$.

3. Schneider, B. (1988) Statistische Klassifikations- und Diskriminanzverfahren. Lab. Med. 12, 69-75.

Prof. Dr. B. Schneider

Institut für Biometrie

Medizinische Hochschule Hannover

Konstanty-Gutschow-Straße 8

D-30625 Hannover

Germany 